\title{
Détermination analytique des paramètres de Potier d'une machine synchrone par la méthode des pentes
}

\author{
Titre court : Calcul des paramètres de Potier \\ Georges L. LOUM (loum_g@yahoo.fr), T. Cissé Haba (cissetheodore@yahoo.fr) \\ Laboratoire d'Electronique et d'Electricité Appliquées \\ Département de formation et de recherche génie électrique et électronique \\ Institut National Polytechnique Houphouët Boigny de Yamoussoukro \\ BP 1093 Yamoussoukro Côte d'Ivoire \\ Auteur correspondant : Georges L. LOUM : loum_g@yahoo.fr
}

Biographies :

Georges Laussane LOUM est ingénieur électrotechnicien de I'Institut National Supérieur de l'Enseignement Technique de Yamoussoukro, Côte d'Ivoire et est docteur en Traitement d'Images de I'université Paris XII Val de Marne. Ses activités pédagogiques et de recherche au Département Génie électrique et Electronique de I'Institut National Polytechnique Houphouët Boigny (INP-HB) de Yamoussoukro (Côte d'Ivoire), concernent les machines électriques et l'analyse d'images de texture.

Théodore Cissé HABA est enseignant chercheur à l'institut National Polytechnique Houphouët Boigny (INP-HB) de Yamoussoukro (Côte d'Ivoire). Diplômé d'un Doctorat de l'université Paul Sabatier de Toulouse III (France) en électronique, il a travaillé sur le comportement fréquentiel de dispositifs microélectroniques à impédance fractionnaire. Aujourd'hui, ses travaux sont orientés vers les traitements numériques et la mise en œuvre de dispositifs électroniques pour des systèmes solaires.

\section{RÉSUMÉ :}

Le modèle de Potier d'une machine synchrone saturée à pôles lisses, est caractérisé par deux paramètres : le coefficient d'équivalence $\alpha$ et la réactance de fuite $\lambda \omega$. De façon classique ces paramètres se déterminent graphiquement à partir de caractéristiques relevées lors de la réalisation de trois essais sur la machine : un essai à vide, un essai en court-circuit et un essai en déwatté. Dans cet article, nous proposons une méthode analytique de détermination des paramètres de Potier. Les expressions de $\alpha$ et de $\lambda \omega$ obtenues, font intervenir les pentes de droites associées aux caractéristiques précédentes. Nous montrons que le calcul de ces pentes est aisé et qu'il nécessite le relevé d'un nombre réduit de points. Les expérimentations effectuées ont confirmé la validité de la méthode proposée.

MOTS-CLÉS : machine synchrone, pôles lisses, Potier, méthode des pentes, réactance de fuite, coefficient d'équivalence, 


\section{Introduction}

Malgré le développement récent de modèles plus élaborés d'étude de la machine synchrone $[1,2,3,4]$, les modèles traditionnels comme ceux de Behn Eschenburg et de Potier continuent de présenter un intérêt certain sur les plans pédagogique et pratique. En effet, ces derniers permettent d'une part, d'introduire plus simplement l'étude de la machine, et d'autre part, de prédéterminer avec une bonne précision, ses conditions de fonctionnement en charge.

Le modèle de base de Potier s'applique à une machine synchrone à pôles lisses pouvant être saturée. Il suppose que le flux magnétique total traversant l'enroulement d'induit, est décomposable en un flux $\phi_{r}$ qui emprunte un circuit saturable (roue polaire, entrefer et carcasse d'induit) et en un flux de fuite $\phi_{f}$ qui se referme essentiellement dans l'air [5].

Au flux de fuite $\phi_{f}$, Potier a associé la réactance de fuite $\lambda \omega$. Il a également suggéré que la force magnétomotrice, responsable du flux $\phi_{r}$, soit décomposée en une force due à la roue polaire et en une autre provenant de la réaction magnétique d'induit [6]. Le coefficient d'équivalence noté $\alpha$ qu'il a défini dans ce cadre, permet de ramener le courant d'induit à l'inducteur.

Classiquement, les paramètres $\alpha$ et $\lambda \omega$ de Potier se déterminent par une méthode graphique basée sur la réalisation d'un essai à vide, d'un essai en court-circuit et d'un essai en déwatté. Parce que graphique, cette méthode est par essence sujette à des erreurs de construction.

Dans cet article, nous proposons une méthode purement analytique de détermination des paramètres de Potier fondée sur les trois essais précédents. Sous certaines conditions de fonctionnement de la machine, les caractéristiques associées à ces essais peuvent être modélisées par des droites. Nous montrons qu'il est alors possible d'exprimer les paramètres de Potier d'une machine synchrone à pôles lisses en fonction des pentes de ces droites.

Dans le paragraphe suivant, nous rappelons brièvement les principales étapes de la méthode de Potier. Puis, nous décrivons la nouvelle méthode de détermination de $\alpha$ et de $\lambda \omega$ (section 3.1), ainsi que les essais à mettre en œuvre pour son application (section 3.2). Enfin, nous présentons les différentes expérimentations (section 4) qui ont été entreprises afin de s'assurer de la validité de la méthode proposée.

\section{Méthode de détermination graphique des paramètres de Potier}

\subsection{Equations générales de Potier}

Les équations générales du modèle de Potier d'une machine synchrone à pôles lisses pouvant être saturée, sont les suivantes :

$\left\{\begin{array}{l}\underline{V}=\underline{E}_{r}-R \underline{I}-j \lambda \omega \underline{I} \\ \underline{J}_{e}=\underline{J}_{e o}-\alpha \underline{I}\end{array}\right.$ 
Dans l'équation aux tensions, $V$ désigne la tension d'une phase de l'alternateur et I le courant d'induit débité. $E_{r}$ traduit la force électromotrice induite par les variations du flux $\phi_{r}$ et $\lambda \omega \mathrm{l}$ représente la chute de tension interne due au flux de fuite $\phi_{\mathrm{f}}$. $\mathrm{R}$ désigne la résistance d'une phase de l'induit de la machine.

L'équation aux intensités établit une relation entre le terme $\alpha$, le courant d'excitation réel Je de la roue polaire et le courant d'excitation fictif $J_{\mathrm{e} 0}$ introduit par Potier. $\mathrm{J}_{\mathrm{e} 0}$ est lié à la force électromotrice induite $E_{r}$, par la caractéristique interne de la machine. Le diagramme vectoriel correspondant au fonctionnement sur charge inductive est donné à la figure 1.a.

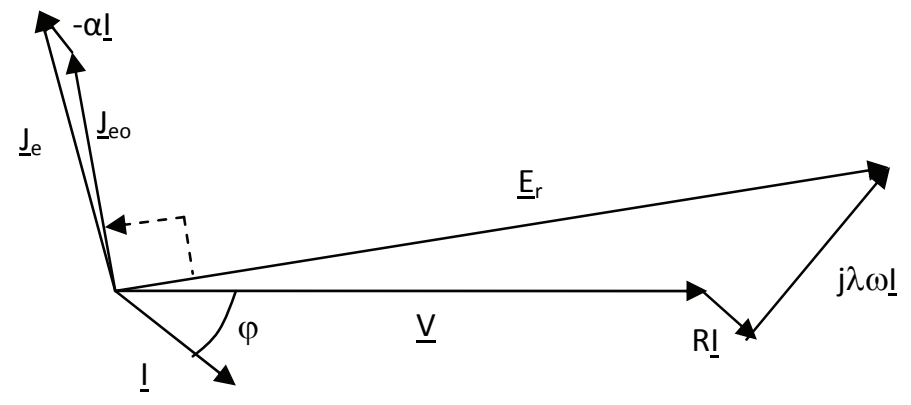

(a)

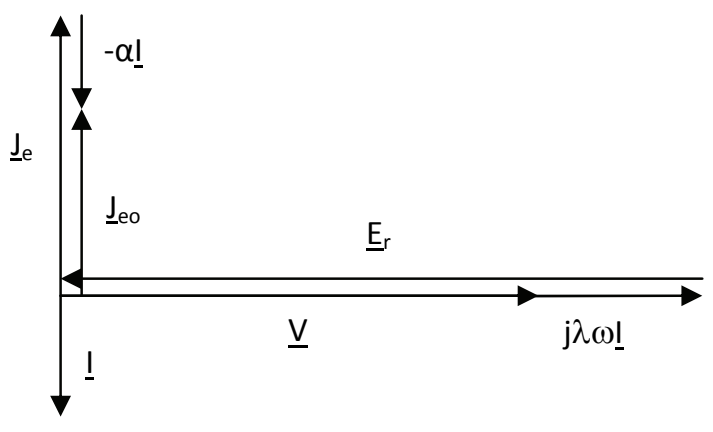

(b)

Figure 1. Diagrammes vectoriels d'une MS débitant sur (a) charge $R-L$,

(b) charge purement inductive

\subsection{Détermination graphique des paramètres de Potier}

La méthode que Potier a proposé pour déterminer les paramètres $\alpha$ et $\lambda \omega$, est purement graphique. Elle est abondamment décrite dans la littérature $[4,5,6,7]$. Trois essais à vitesse de rotation constante, sont nécessaires à sa mise en œuvre :

- Un essai à vide qui permet de tracer la caractéristique interne $E_{r}=f\left(J_{e 0}\right)$,

- Un essai en déwatté relevé à courant d'induit $\mathrm{I}_{1}$ et permettant d'obtenir le point $H\left(\mathrm{~J}_{\mathrm{e}}, \mathrm{V}_{1}\right)$,

- Un essai en court-circuit qui permet de placer le point C. Celui-ci est relevé pour un courant d'excitation $J_{\text {ecc } 1}$ tel que le courant de court-circuit $I_{c c}$ soit égal au courant d'induit $I_{1}$ de l'essai en déwatté.

Traditionnellement, l'essai en déwatté est réalisé avec une charge purement inductive. Cependant celle-ci peut être remplacée par une charge purement capacitive [6].

Pour un essai en déwatté sur charge inductive, réalisé autour du point nominal, la chute de tension RI peut être considérée comme négligeable devant $V$ et les équations vectorielles de (1) peuvent se ramener alors aux équations scalaires (2) ci-dessous. Le diagramme vectoriel relatif à ce fonctionnement est donné à la figure 1.b.

$\left\{\begin{array}{l}V=E_{r}-\lambda \omega I \\ J_{e}=J_{e o}+\alpha I\end{array}\right.$ 
Les équations (2) indiquent que lorsque la charge est purement inductive, la courbe $V=f\left(\mathrm{~J}_{\mathrm{e}}\right)$ peut être déduite de la caractéristique interne $E_{r}=f\left(J_{e 0}\right)$, par une translation de composantes $(-\alpha \mathrm{l} ; \lambda \omega \mathrm{l})$. Cette translation associe à tout point $\mathrm{H}$ de la caractéristique $\mathrm{V}=\mathrm{f}\left(\mathrm{J}_{\mathrm{e}}\right)$, un point $\mathrm{M}$ de la caractéristique interne. En particulier, le point $C$ de court-circuit de la figure 2 qui appartient à la caractéristique $\mathrm{V}=\mathrm{f}\left(\mathrm{J}_{\mathrm{e}}\right)$, a pour image un point $\mathrm{B}$ de position exacte inconnue, mais d'appartenance certaine à la partie linéaire de la caractéristique interne.

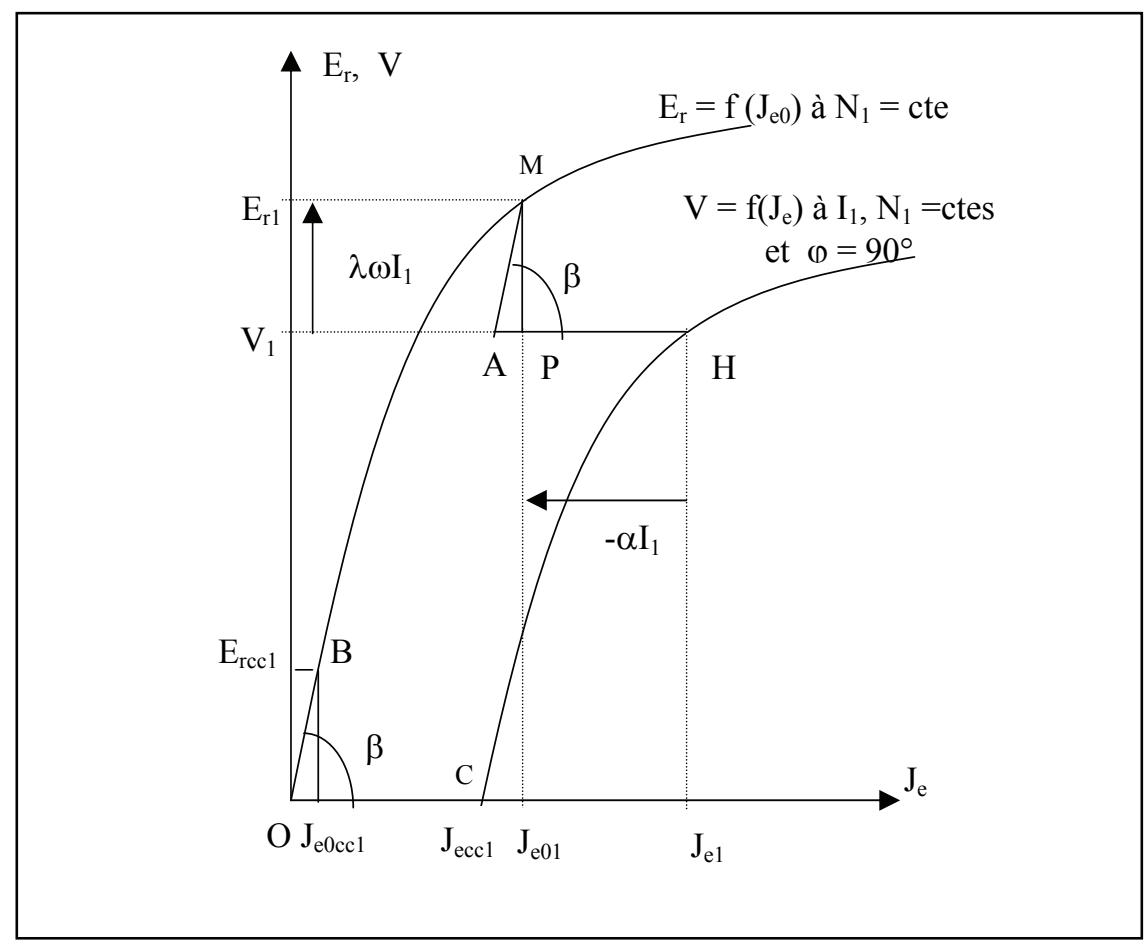

Figure 2. Méthode de détermination graphique des paramètres de Potier

Il est alors possible de définir l'angle $\beta$ et la distance $\mathrm{OC}=\mathrm{J}_{\mathrm{ecc} 1}$ qui sont les caractéristiques connues du triangle COB. Les dimensions de ce triangle à l'instar des composantes de la translation, sont constantes pour un courant $d^{\prime}$ induit $l=l_{1}$ et une vitesse de rotation donnés. De ce fait, l'image $M$ d'un point $H$ de la caractéristique $V=f\left(J_{e}\right)$, peut être obtenue en construisant le triangle HAM d'angle $\beta$ en $A$, similaire au triangle COB.

En appliquant ce résultat au point relevé lors de l'essai en déwatté, les composantes de la translation, $\mathrm{HP}=\left(-\left.\alpha\right|_{1}\right)$ et $\mathrm{MP}=\left(\lambda \omega \mathrm{I}_{1}\right)$, peuvent être mesurées et les valeurs de $\alpha$ et de $\lambda \omega$ déduites de ces mesures :

$\lambda \omega=\frac{M P}{I_{1}} ; \alpha=\frac{P H}{I_{1}}$

Comme toute méthode graphique, celle que nous venons de décrire, est sensible aux erreurs de construction. Pour s'affranchir de ce type d'erreurs, nous proposons dans ce qui suit, une nouvelle méthode de détermination des paramètres de Potier qui a l'avantage d'être entièrement analytique.

\section{Détermination analytique des paramètres de Potier par la méthode des pentes}




\subsection{Expressions analytiques des paramètres $\alpha$ et $\lambda \omega$}

Considérons l'équation aux tensions de (1) (section 2.1). Si la machine synchrone, normalement excitée, débite sur une charge purement inductive, le terme RI peut être négligé devant $V$ et nous obtenons l'équation aux tensions de (2) de Potier (section 2.2). En dérivant cette équation par rapport au courant d'induit I, nous obtenons I'expression cidessous:

$\frac{d E_{r}}{d I}=\frac{d V}{d I}+\lambda \omega$

Cette expression montre que la réactance $\lambda \omega$ peut être déterminée à partir de la connaissance des dérivées par rapport à I de $E_{r}$ et de $V$.

\subsubsection{Expression de la dérivée de $E_{r}$ par rapport à I}

Si nous nous plaçons en un point de fonctionnement proche du court-circuit $(\mathrm{V} \rightarrow 0)$, la tension $\mathrm{V}$ peut être considérée comme négligeable devant la chute de tension due à l'impédance interne de la machine. L'équation aux tensions de (1) (section 2.1) nous permet alors d'écrire :

$\left(\underline{E}_{r}\right)_{V \rightarrow 0}=(R+j \lambda \omega) \times(\underline{I})_{V \rightarrow 0} \Rightarrow\left(E_{r}^{2}\right)_{V \rightarrow 0}=\left(R^{2}+(\lambda \omega)^{2}\right) \times\left(I^{2}\right)_{V \rightarrow 0}$

La dérivée de l'équation (5) par rapport à $\mathrm{I}$, donne une expression du rapport $\mathrm{dE}_{\mathrm{r}} / \mathrm{dl}$ en fonction de $\mathrm{R}$ et de $\lambda \omega$ relative à un fonctionnement en court-circuit :

$\left(\frac{d E_{r}}{d I}\right)_{V \rightarrow 0}= \pm \sqrt{R^{2}+(\lambda \omega)^{2}}=$ constante

Or en court-circuit, la machine n'a pratiquement pas de flux et n'est donc pas saturée. La formule (6) qui a été établie pour un fonctionnement proche du court-circuit, n'est donc pas directement applicable à un fonctionnement normal où la machine se trouve dans un état magnétique saturé. Aussi convient-il de rechercher la formule équivalente à (6) dans le cas d'une machine saturée, hypothèse d'étude de Potier.

Le modèle proposé par Potier est d'autant valide que le courant d'excitation $\mathrm{J}_{\mathrm{e} 1}$ est situé dans la zone de saturation [6], c'est-à-dire comme le montre la figure 3, au-delà du coude de la caractéristique interne de la machine. A ce courant d'excitation correspond la force électromotrice à vide $\mathrm{E}_{\mathrm{r} 01}$. 
Figure 3. Caractéristique interne d'une machine synchrone

Dans le cas d'une charge purement inductive, le passage du fonctionnement à vide $\left(\mathrm{J}_{\mathrm{e} O \mathrm{v}}=\mathrm{J}_{\mathrm{e} 1}\right)$ au fonctionnement en charge $\left(\mathrm{J}_{\mathrm{e} 0 \mathrm{ch}}=\mathrm{J}_{\mathrm{e} 1}-\alpha \mathrm{l}\right)$, se traduit par une réduction de $\alpha \mathrm{l} d \mathrm{~d}$ courant d'excitation $\mathrm{J}_{\mathrm{e} 1}$ et partant, par une baisse de la force électromotrice qui passe de $\mathrm{E}_{\mathrm{r} 01}$ à $\mathrm{E}_{\mathrm{r} 02}$.

Cette variation de la force électromotrice, peut être considérée comme linéaire si le courant d'induit I est faible $(I \rightarrow 0)$. L'état magnétique de la machine évolue alors dans un domaine restreint de la zone de saturation, que nous avons qualifié de zone linéarisée sur la figure 3. Désignons par (D1) la droite de pente $\mathrm{k}_{\text {lin }}$ qui modélise la zone linéaire de la caractéristique interne de la figure 2. Dans notre approche, cette droite est associée au fonctionnement proche du court-circuit pour lequel la machine est non saturée. Nommons (D2), la droite de pente $\mathrm{k}_{\text {sat }}$ de la zone saturée linéarisée qui est rattachée au fonctionnement en charge, à faible débit de courant $(I \rightarrow 0)$. L'équation aux courants de (2), permet alors d'établir une relation linéaire entre $E_{\mathrm{r}}$ et $I$ dans les deux cas de fonctionnement considérés :

$\left\{\begin{array}{l}\left(E_{r}\right)_{V \rightarrow 0}=k_{\text {lin }} \times J_{e 0}=k_{\text {lin }}\left(J_{e 1}-\alpha(I)_{V \rightarrow 0}\right) \\ \left(E_{r}\right)_{I \rightarrow 0}=k_{\text {sat }} \times J_{e 0}+b=k_{\text {sat }}\left(J_{e 1}-\alpha(I)_{I \rightarrow 0}\right)+b\end{array}\right.$

Le coefficient $b$ et les pentes $k_{\text {lin }}$ et $k_{\text {sat }}$ des droites (D1) et (D2) sont constants pour une vitesse de rotation donnée. Aussi, à vitesse et à courant d'excitation $\mathrm{J}_{\mathrm{e} 1}$ constants, si nous dérivons $E_{r}$ par rapport à I dans (7), nous obtenons les équations (8) ci-dessous relatives aux fonctionnements en court-circuit (8.1) et à faible courant de charge (8.2) :

$\left\{\begin{array}{l}\left(\frac{d E_{r}}{d I}\right)_{V \rightarrow 0}=-\alpha \times k_{\text {lin }} \\ \left(\frac{d E_{r}}{d I}\right)_{I \rightarrow 0}=-\alpha \times k_{\text {sat }}\end{array}\right.$

Le rapport des deux équations conduit à :

$\left(\frac{d E_{r}}{d I}\right)_{I \rightarrow 0}=\frac{k_{\text {sat }}}{k_{\text {lin }}} \times\left(\frac{d E_{r}}{d I}\right)_{V \rightarrow 0}=\eta \times\left(\frac{d E_{r}}{d I}\right)_{V \rightarrow 0} \quad$ avec $\quad \eta=\frac{k_{\text {sat }}}{k_{\text {lin }}}$

Ainsi, pour qu'elle puisse être également valable en charge, l'expression (6) du rapport $\mathrm{dEr} / \mathrm{dl}$ établie en court-circuit, doit être multipliée dans notre approche, par un coefficient $\eta$ qui est le rapport des pentes des droites (D2) et (D1) modélisant les zones linéarisée et linéaire de la caractéristique interne de la machine. Notons que du fait de la saturation, le coefficient $\eta$ sera toujours nettement inférieur à 1.

\subsubsection{Expression de la dérivée de V par rapport à I}


Rappelons que notre approche se fonde sur les conditions suivantes:

- La machine est saturée (état magnétique au-delà du coude de saturation)

- Un fonctionnement sur charge purement inductive, à faible courant d'induit, pour que l'état magnétique de la machine se situe dans la zone linéarisée et que I'hypothèse d'une force électromotrice $E_{r}$, variant linéairement avec le courant débité $\mathrm{l}$, soit vérifiée.

Aussi, sous ces conditions et en se référant à l'équation aux tensions de (2) (section 2.2), la caractéristique $\mathrm{V}=\mathrm{f}(\mathrm{I})$ obtenue pour de faibles courants d'induit, est une droite. La dérivée est donc égale à une différence et le rapport $\mathrm{dV} / \mathrm{dl}$ est la pente $\mathrm{k}_{\mathrm{ch}}$ à l'origine de la caractéristique en charge $\mathrm{V}=\mathrm{f}(\mathrm{I})$ relevée à courant $\mathrm{d}$ 'excitation et à vitesse constants :

$k_{c h}=\left(\frac{d V}{d I}\right)_{I \rightarrow 0}=\left(\frac{\Delta V}{\Delta I}\right)_{I \rightarrow 0}$

Notons que $k_{c h}$ est négatif à cause de l'effet démagnétisant de la réaction magnétique d'induit de la machine sur charge inductive.

\subsubsection{Expression de la réactance $\lambda \omega$ de Potier}

Les relations (8.1) et (8.2) (section 3.1.1) indiquent que le rapport $\mathrm{dE}_{\mathrm{r}} / \mathrm{dl}$ est négatif quel que soit le fonctionnement considéré. Aussi, en intégrant les relations (6), (9) et (10) dans (4) (section 3.1), nous obtenons l'équation en $\lambda \omega$ suivante :

$-\eta \sqrt{R^{2}+(\lambda \omega)^{2}}=k_{c h}+\lambda \omega$

Le rapport $\mathrm{dE}_{\mathrm{r}} / \mathrm{dl}$ étant également négatif, il découle de l'équation 4 (section 3.1 ) que la réactance $\lambda \omega$ est toujours inférieure à la valeur absolue de $\mathrm{dV} / \mathrm{dl} c^{\prime}$ est-à-dire à celle de $\mathrm{k}_{\mathrm{ch}}$.

Ainsi, la résolution de l'équation (11) conduit à l'expression analytique (12) de la réactance $\lambda \omega$ de Potier qui dépend des pentes $k_{\text {lin }}, k_{c h}$ et $k_{\text {sat }}$ et de la résistance statorique $R$.

$\lambda \omega=\frac{\left|k_{c h}\right|-\eta \sqrt{k_{c h}^{2}+R^{2}\left(1-\eta^{2}\right)}}{1-\eta^{2}}=\frac{\left.\left|k_{c k}\right|-\frac{k_{\text {sat }}}{k_{\text {lin }}} \sqrt{\left(k_{c h}\right)^{2}+R^{2}\left(1-\left(\frac{k_{\text {sat }}}{k_{\text {lin }}}\right)^{2}\right.}\right)}{1-\left(\frac{k_{\text {sat }}}{k_{\text {lin }}}\right)^{2}}$

\subsubsection{Expression du coefficient d'équivalence $\alpha$ de Potier}

La détermination de l'expression analytique du coefficient d'équivalence $\alpha$ est fondée sur la réalisation d'un essai en court-circuit. Pour ce fonctionnement, l'expression de $\alpha$ se déduit de l'équation aux courants de (2) ( section 2.2) :

$\alpha=\frac{1}{I_{c c 1}}\left(J_{e c c 1}-J_{e 0 c c 1}\right)$

Le calcul de $J_{\text {ecc1 } 1}$ et de $J_{\text {eocc } 1}$ s'effectue simplement en supposant que le courant de courtcircuit $I_{\mathrm{cc} 1}$ est égal au courant I de l'essai en déwatté. En effet, l'équation (5) ( section 3.1.1) permet alors de calculer $E_{r c c 1}$ puis d'en déduire $J_{e 0 c c 1}=E_{r c c 1} / k_{\text {lin }}$ (figure 1). D'un autre côté, il 
est connu que la caractéristique en court-circuit $I_{c c}=f\left(J_{e}\right)$ de la machine synchrone est une droite linéaire. Le rapport $I_{c c 1} / J_{\text {ecc1 }}$ correspond à la pente $k_{c c}$ de cette droite. Ce faisant, l'expression analytique de $\alpha$ est :

$\alpha=\frac{1}{k_{c c}}-\frac{\sqrt{R^{2}+(\lambda \omega)^{2}}}{k_{\text {lin }}}$

Notons que les pentes $k_{\text {ch }}$ et $k_{\text {sat }} n$ 'interviennent pas dans les formules de $\alpha$. Ce qui est somme toute logique puisque lors de l'essai en court-circuit, la machine n'est ni chargée, ni saturée.

\subsubsection{Expressions simplifiées des paramètres de Potier}

Les expressions analytiques (12) (section 3.1.3) et (14) de $\lambda \omega$ et de $\alpha$ nous paraissent assez lourdes. Des formules beaucoup plus simples peuvent être obtenues sous certaines hypothèses généralement admises : résistance statorique $R$ négligée et machine fortement saturée $(\eta<1)$.

Ces formules simplifiées qui sont facilement exploitables et qui font intervenir les différentes pentes que nous avons définies, sont regroupées dans la table 1.

Table 1 : Différentes expressions des paramètres de Potier

\begin{tabular}{|c|c|c|c|}
\hline $\mathbf{N}^{\circ}$ & Hyp. & Expression de $\lambda \omega$ & Expression de $\alpha$ \\
\hline$(15.1)$ & Aucune & $(\lambda \omega)=\frac{\left|k_{c k}\right|-\eta \sqrt{\left(k_{c h}\right)^{2}+R^{2}\left(1-\eta^{2}\right)}}{1-\eta^{2}}$ avec $\eta=\frac{k_{\text {sat }}}{k_{\text {lin }}}$ & $\alpha=\frac{1}{k_{c c}}-\frac{\sqrt{R^{2}+(\lambda \omega)^{2}}}{k_{\text {lin }}}$ \\
\hline$(15.2)$ & $\mathrm{R} \approx 0$ & $\lambda \omega=\frac{\left|k_{c k}\right|}{1+\eta}$ avec $\eta=\frac{k_{s a t}}{k_{\text {lin }}}$ & $\alpha=\frac{1}{k_{c c}}-\frac{\lambda \omega}{k_{\text {lin }}}$ \\
\hline$(15.3)$ & $\begin{array}{l}\mathrm{R} \approx 0 \text { et } \\
\eta<<1\end{array}$ & $\lambda \omega=\left|k_{c h}\right|$ & $\alpha=\frac{1}{k_{c c}}-\frac{\left|k_{c h}\right|}{k_{\text {lin }}}$ \\
\hline
\end{tabular}

Les formules (15.3) indiquent que les paramètres de Potier peuvent en première approximation, s'exprimer en fonction de trois des quatre pentes que nous avons définies à savoir, $k_{c h}, k_{\text {lin }}$ et $k_{c c}$. Il est également intéressant de faire remarquer que les formules (15.2) et (15.3) établissent une relation linéaire entre les deux paramètres de Potier $\alpha$ et $\lambda \omega$. Ces deux grandeurs évoluent en sens inverse.

\subsection{Essais à mettre en œuvre}

La méthode que nous venons d'exposer n'impose aucun tracé de caractéristiques pour la détermination des paramètres de Potier. Les essais à mettre en œuvre doivent permettre de calculer les valeurs des différentes pentes résumées ci-dessous et qui interviennent dans les formules que nous avons établies. Ce sont :

- $\quad k_{\text {lin }}$ : pente de la partie linéaire de la caractéristique à vide de la machine

- $\quad \mathrm{k}_{\text {sat }}$ : pente de la zone de saturation linéarisée de la caractéristique à vide de la machine

- $\quad k_{c h}$ : pente à l'origine de la caractéristique en charge de la machine débitant sur une charge purement inductive 
- $\quad k_{c c}$ : pente de la caractéristique en court-circuit de la machine.

Pour déterminer ces pentes, il faut un minimum de cinq points relevés au cours de trois essais réalisés à vitesse de rotation constante. Ces points représentés sur la figure 4, appartiennent aux caractéristiques à vide, en court-circuit et en charge de la machine. Ainsi, - L'essai à vide fournit trois points :

- Le premier point P1 est utilisé pour calculer $k_{\text {lin }}$. Il doit correspondre à un courant d'excitation assez faible pour être sûr que la machine reste dans un état magnétique non saturé.

$$
k_{\text {lin }}=\frac{E_{r 0}(P 1)}{J_{e 0}(P 1)}
$$

- Les deuxième et troisième points ( $P 2$ et $P 3)$ qui permettent de déterminer $k_{\text {sat }}$, seront pris dans la zone linéarisée (zone de saturation). Les valeurs des courants d'excitation de ces points doivent par conséquent être proches de la valeur nominale et leur écart doit être faible. Les paramètres de Potier seront déterminés pour le point P3 qui possède le courant d'excitation le plus élevé. Dans notre approche, ce courant d'excitation est noté $J_{\mathrm{e} 1}$. Précisons que le point P3 est également un point de la caractéristique en charge.

$$
k_{s a t}=\frac{E_{r 0}(P 3)-E_{r 0}(P 2)}{J_{e 1}-J_{e 0}(P 2)}
$$

- L'essai en déwatté à faible courant de charge $I_{1}$ permet de relever un autre point (P4) de la caractéristique en charge. $\mathrm{P} 4$ et le point à vide précédent $\mathrm{P} 3$, ont tous deux le même courant $d^{\prime}$ excitation $\mathrm{J}_{\mathrm{e} 1}$. Ils seront utilisés pour déterminer $\mathrm{k}_{\mathrm{ch}}$.

$$
k_{c h}=\frac{E_{r 0}(P 4)-E_{r 0}(P 3)}{I(P 4)}
$$

- L'essai en court-circuit fournit le cinquième et dernier point (P5) nécessaire à la mise en œuvre de la méthode. Ce point sert à calculer $k_{c c}$. La caractéristique $I_{c c}=f\left(J_{e}\right)$ étant connue linéaire, nous pouvons relever n'importe quel point de cette caractéristique dont en particulier, celui de courant de court-circuit égal au courant de charge $I_{1}$ de l'essai en déwatté.

$$
k_{c c}=\frac{I_{c c}(P 5)}{J_{e}(P 5)}
$$




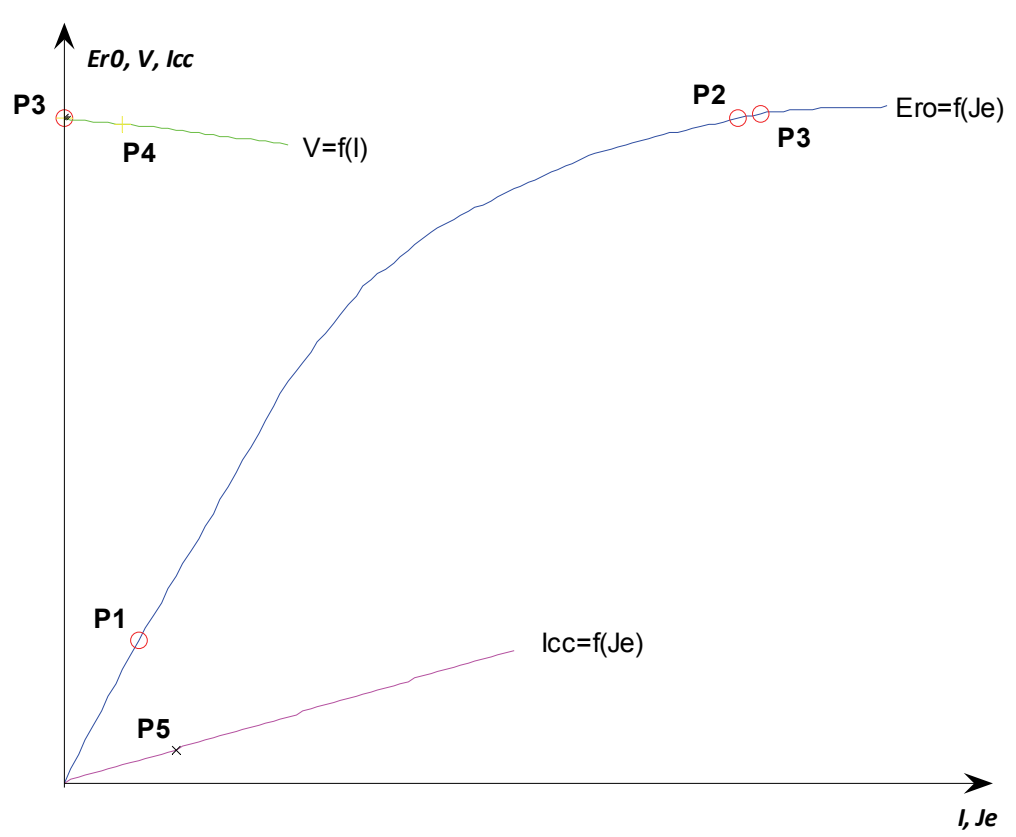

Figure 4. Points nécessaires à la mise en œuvre de la méthode analytique

Pour conclure sur les essais, il est important d'indiquer que la méthode graphique traditionnelle et celle que nous présentons dans cet article, nécessitent toutes deux la connaissance d'au moins un point de l'essai en déwatté. Cependant, la caractéristique utilisée pour la détermination des paramètres de Potier diffère selon la méthode. La méthode classique est basée sur la caractéristique $V=f(J e)$ à courant d'induit constant, tandis que dans notre méthode, c'est la caractéristique en charge $V=f(I)$ à courant d'excitation constant qui est employée.

\section{Expérimentations}

\subsection{Expérimentations réalisées sur la machine MS1}

Les premiers essais nécessaires à la validation de notre méthode ont été réalisés sur une machine synchrone triphasée notée MS1, de caractéristiques nominales :

$3 \mathrm{KVA} ; 220 / 380 \mathrm{~V} ; 8 / 4,5 \mathrm{~A} ; \cos \varphi=0,8 ; 1500 \mathrm{tr} / \mathrm{mn} ; 50 \mathrm{~Hz}$; Excitation : 9,5 A.

La caractéristique à vide relevée à 1500 tr/mn a donné les valeurs de la table 2 .

Table 2 : Valeurs relevées lors de l'essai à vide de MS1

\begin{tabular}{|l|c|c|c|c|c|c|c|c|c|c|c|c|}
\hline $\mathrm{E}_{\mathrm{r}}(\mathrm{V})$ & 0 & 68 & 125 & 175 & 213.5 & 237 & 250 & 261 & 270 & 272 & 277 & 279 \\
\hline $\mathrm{J}_{\mathrm{e} 0}(\mathrm{~A})$ & 0 & 1 & 2 & 3 & 4 & 5 & 6 & 7 & 8 & 8.3 & 9 & 9,3 \\
\hline
\end{tabular}

La table 3 renferme les valeurs de l'essai en déwatté sur charge inductive à $1500 \mathrm{tr} / \mathrm{mn}$ et à courant d'excitation constant $\mathrm{J}_{\mathrm{e} 1}=8,3 \mathrm{~A}$.

Table 3 : Valeurs relevées lors de l'essai en déwatté de MS1 


\begin{tabular}{|l|c|c|c|c|c|c|c|c|}
\hline $\mathrm{V}(\mathrm{V})$ & 272 & 270,5 & 268,5 & 265 & 263,5 & 242 & 198 & 168 \\
\hline $\mathrm{I}(\mathrm{A})$ & 0 & 0,18 & 0,4 & 0,76 & 0,9 & 2 & 3,5 & 4,3 \\
\hline
\end{tabular}

La caractéristique qui a été relevée lors de l'essai en court circuit est représentée à la figure $5 a$. Son équation est de la forme : $I_{c c}=0,833 \times J_{e}$. Nous avons également représenté à la figure $5 b$, la caractéristique à faible charge pour un régime déwatté, afin de s'assurer qu'elle était linéaire comme nous l'avons affirmé. La courbe obtenue confirme nos résultats théoriques.

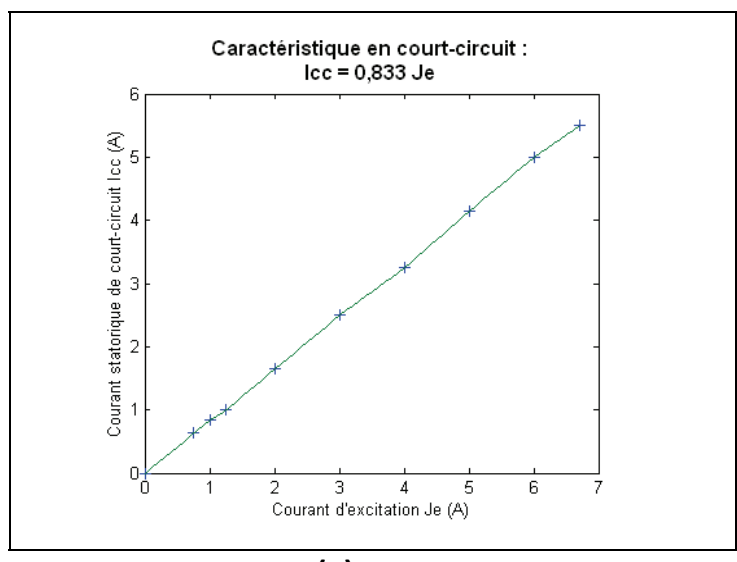

(a)

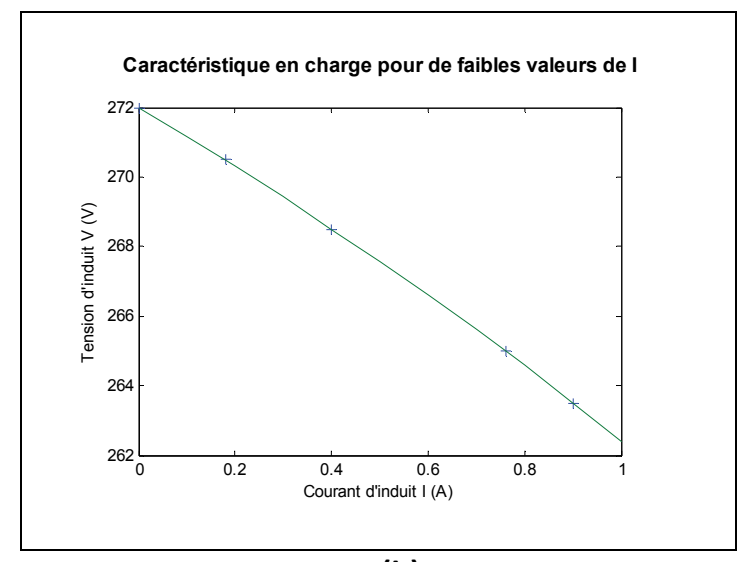

(b)

Figure 5. Caractéristiques de MS1 (a) en court-circuit et (b) à faible charge en régime déwatté

La mesure à chaud de la résistance de l'induit a donné une valeur $\mathrm{R}$ égale à $2,3 \Omega$.

Les paramètres de Potier ont été déterminés graphiquement par la méthode classique pour un courant d'excitation $\mathrm{J}_{\mathrm{e} 1}$ égal à $8,3 \mathrm{~A}$. Pour réduire les erreurs de construction, la détermination graphique, visible à la figure 6 , a été réalisée sous Matlab pour le point de fonctionnement $(I=4,3 \mathrm{~A} ; \mathrm{V}=165 \mathrm{~V})$ de l'essai en déwatté. Nous avons obtenu comme valeurs de paramètres : $\lambda \omega=7,3427 \Omega$ et $\alpha=1,0863$.

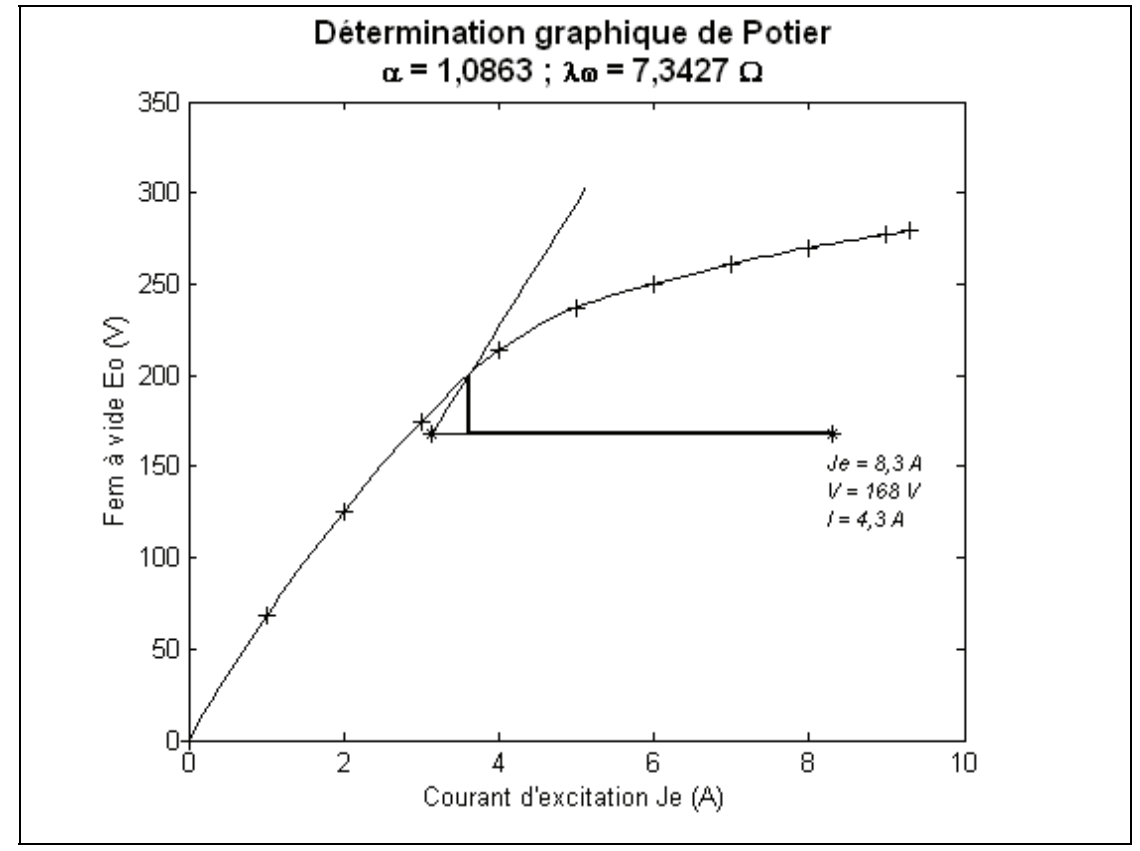


Figure 6. Détermination graphique des paramètres de Potier de MS1

Pour s'assurer de la validité de la méthode proposée, nous avons déterminé par les formules exactes (15.1) de la table 1, les valeurs des paramètres de Potier pour le même courant $d^{\prime}$ excitation $\mathrm{J}_{\mathrm{e} 1}=8,3 \mathrm{~A}$. Ces valeurs ont été calculées pour des courants d'induit n'excédant pas $20 \%$ de la valeur nominale. De plus, conformément à notre approche, la valeur de la pente $\mathrm{k}_{\text {sat, }}$ de la caractéristique interne, a été calculée pour une faible variation $\mathrm{du}$ courant d'excitation $\left(\mathrm{J}_{\mathrm{e} 0 \mathrm{ch}}-\mathrm{J}_{\mathrm{e} 1}=8-8,3=-0,3 \mathrm{~A}\right)$ se traduisant par une chute de tension de $2 \mathrm{~V}$ de la force électromotrice.

Les résultats obtenus et regroupés dans la table 4 indiquent que les valeurs calculées par notre méthode sont du même ordre de grandeur que celles fournies par la méthode graphique. De plus, conformément à l'approche adoptée, l'écart entre les valeurs fournies par les deux méthodes est d'autant réduit que le courant d'induit est faible.

Table 4 : Valeurs des paramètres de Potier de MS1 fournies par la méthode analytique

\begin{tabular}{|l|l|c|c|c|}
\cline { 2 - 5 } \multicolumn{1}{c|}{} & $\mathrm{I}(\mathrm{A})$ & $\mathbf{0 , 1 8}$ & $\mathbf{0 , 4}$ & $\mathbf{0 , 7 6}$ \\
\hline \multirow{4}{*}{ Pentes } & $\mathrm{K}_{\text {lin }}$ & \multicolumn{3}{|c|}{68} \\
\cline { 2 - 5 } & $\mathrm{K}_{\mathrm{cc}}$ & \multicolumn{3}{|c|}{0,833} \\
\cline { 2 - 5 } & $\mathrm{K}_{\mathrm{sat}}$ & 6,6667 & $-9,2105$ \\
\cline { 2 - 5 } & $\mathrm{K}_{\mathrm{ch}}$ & $-8,333$ & $-8,750$ & 8.3604 \\
\hline \multirow{3}{*}{ Paramètres } & $\lambda \omega(\Omega)$ & 7.5587 & 7.9396 & 1.0725 \\
\cline { 2 - 5 } & $\alpha$ & 1.0838 & 1.0784 & \\
\hline
\end{tabular}

Pour évaluer la précision de la méthode proposée, nous avons conduit à la vitesse nominale de $1500 \mathrm{tr} / \mathrm{mn}$, différents essais directs, à demi et à pleine charge sur des plans de charge de type inductif, résistif et capacitif. Les valeurs relevées lors de ces essais ont été consignées dans la table 5.

Table 5 : Relevé des essais réalisés sur MS1 pour différents types de charge

\begin{tabular}{|l|c|c|c|c|c|c|}
\hline & \multicolumn{2}{|c|}{ Charge inductive } & \multicolumn{2}{c|}{ Charge résistive } & \multicolumn{2}{c|}{ Charge capacitive } \\
\hline $\cos \varphi$ & 0,8 & 0,78 & 1 & 1 & 0,88 & 0,8 \\
\hline V (V) & 220 & 220 & 220 & 220 & 220 & 220 \\
\hline I (A) & 2,3 & 4,2 & 2,3 & 4,4 & 2,25 & 4,5 \\
\hline Je1 (A) & 6,6 & 9,3 & 5,1 & 7,1 & 3,8 & 4,7 \\
\hline
\end{tabular}

Comme le montre la figure 7 , ces données ont permis de simuler le modèle complet de Potier de la machine sous Matlab. Nous avons dans un premier temps, déterminer les valeurs de $\alpha$ et de $\lambda \omega$ qui minimisent l'erreur entre les valeurs mesurées et celles calculées. Ensuite, nous avons tracé les courbes d'excitation de la machine. 


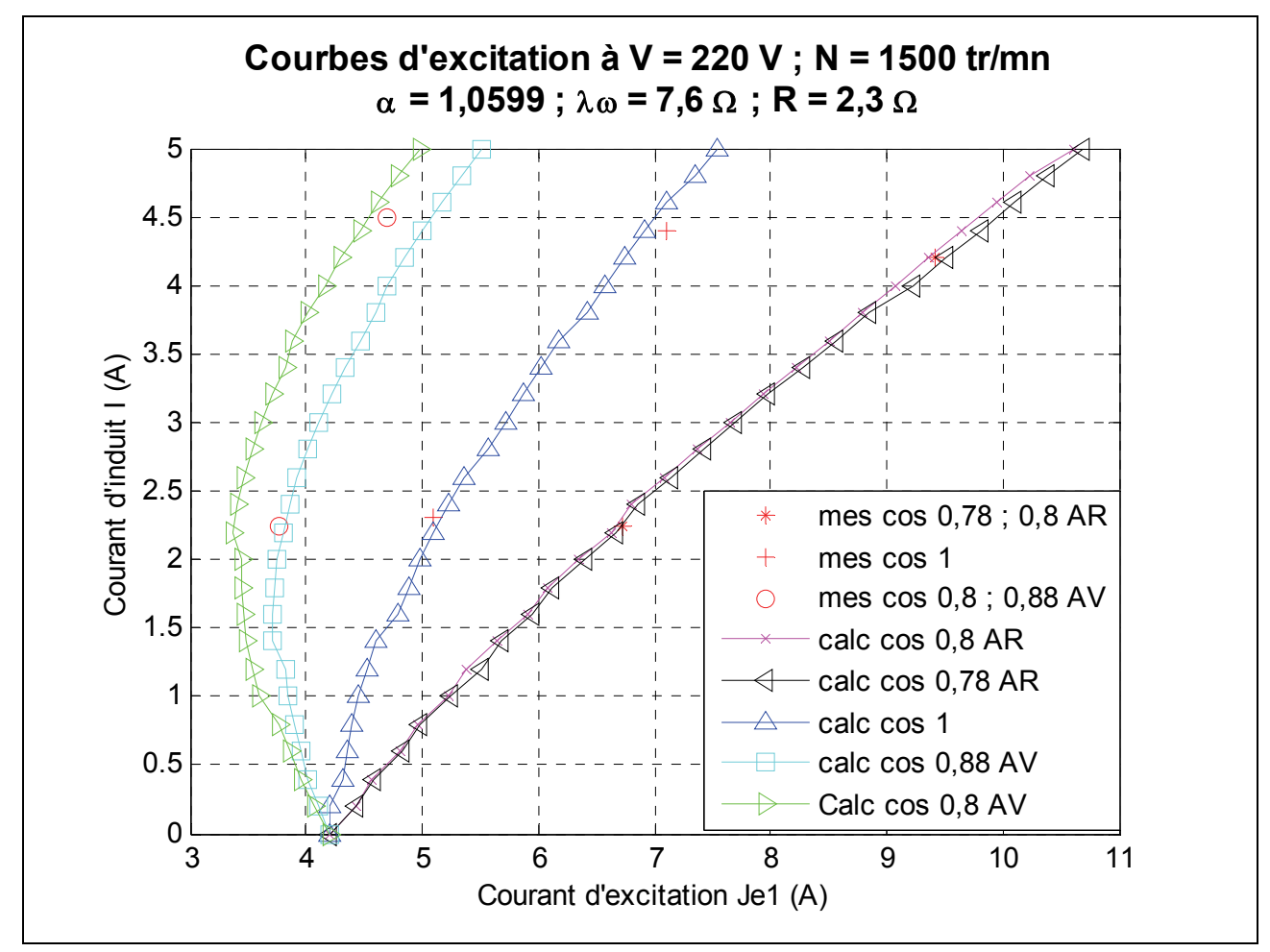

Figure 7. Courbes d'excitation de la machine MS1

Après simulation, nous avons obtenu : $\lambda \omega=7,6 \Omega$ et $\alpha=1,0599$ pour $R=2,3 \Omega$ et $\lambda \omega=8,0 \Omega$ et $\alpha=1,1471$ avec $R=0 \Omega$.

Les valeurs des paramètres de Potier de la table 4, calculées par notre méthode pour le courant d'induit $\mathrm{I}=0,18 \mathrm{~A}$, ont été mises en correspondance dans la table 6 avec les valeurs fournies par la méthode graphique et celles issues des différentes formules simplifiées que nous avons établies (table 1).

Table 6 : Valeurs des paramètres de Potier de MS1 fournies par les différentes méthodes

\begin{tabular}{|l|c|c|c|c|c|}
\cline { 2 - 6 } \multicolumn{1}{c|}{} & \multirow{2}{*}{ Simulation } & $\begin{array}{l}\text { Méthode } \\
\text { Classique }\end{array}$ & \multicolumn{3}{c|}{ Nouvelle méthode (I=0,18A) } \\
\hline Formules & & & $\mathbf{( 1 5 . 1 )}$ & $\mathbf{( 1 5 . 2 )}$ & $\mathbf{( 1 5 . 3 )}$ \\
\hline$\lambda \omega(\Omega)$ & 7,6 & 7,3427 & 7.5587 & 7.5893 & 8.333 \\
\hline$\alpha$ & 1,0599 & 1,0863 & 1.0838 & 1.0884 & 1.0775 \\
\hline$\varepsilon_{\mathrm{R} \lambda \omega}(\%)$ & $0 \%$ & $3,38 \%$ & $0,54 \%$ & $0,14 \%$ & $-6,68 \%$ \\
\hline $\boldsymbol{\varepsilon}_{\mathrm{R} \alpha}(\%)$ & $0 \%$ & $-2,49 \%$ & $-2,20 \%$ & $-2,69 \%$ & $-1,53 \%$ \\
\hline
\end{tabular}

Les valeurs obtenues lors de simulation, pour $\mathrm{R}=2,3 \Omega$, ont été prises comme référence. Les erreurs relatives calculées, indiquent que la précision de notre méthode (formules 15.1) est meilleure à celle de la méthode classique.

Nous pouvons également constater qu'en adoptant les formules simplifiées (15.2 et 15.3) de la table 1, l'erreur commise reste en valeur absolue inférieure à $7 \%$. En particulier, les formules (15.2) constituent une bonne approximation des formules exactes (15.1). Nous les 
adopterons dans la suite pour calculer les valeurs des paramètres de Potier. Les formules (15.3) d'expression beaucoup plus simples, conduisent malheureusement à des erreurs relatives plus importantes. Nous suggérons de les utiliser uniquement, pour déterminer l'ordre de grandeur de $\alpha$ et de $\lambda \omega$.

\subsubsection{Expérimentations réalisées sur la machine MS2}

Une autre série d'expérimentations a été entreprise sur une machine synchrone MS2 de 6 $\mathrm{KVA}, 220 \mathrm{~V}, 50 \mathrm{~Hz}, 1500 \mathrm{tr} / \mathrm{mn}$. Les essais à vide et en court-circuit à la vitesse nominale ont donné les valeurs de la table 7 .

Table 7 : Valeurs relevées lors des essais à vide et en court-circuit de MS2

\begin{tabular}{|l|c|c|c|c|c|c|c|c|c|c|c|c|}
\hline $\mathrm{E}_{\mathrm{r}}(\mathrm{V})$ & 0 & 56 & 78 & 98 & 124 & 138 & 146 & 153 & 159 & 161 & 164 & 169 \\
\hline $\mathrm{J}_{\mathrm{e} 0}(\mathrm{~A})$ & 0 & 1 & 1,5 & 2 & 3 & 4 & 5 & 6 & 7 & 7,5 & 8 & 9 \\
\hline $\mathrm{Icc}(\mathrm{A})$ & 0 & 2.6 & & 5.3 & 7.2 & 10.5 & 13.4 & 16 & & & & \\
\hline
\end{tabular}

Un essai en déwatté à $1500 \mathrm{tr} / \mathrm{mn}$ avec un courant d'excitation constant de $8 \mathrm{~A}$, a été réalisé sur la machine, afin de déterminer les valeurs des paramètres de Potier pour chacune des méthodes étudiées. Les valeurs obtenues sont consignées dans la table 8.

Table 8 : Valeurs des paramètres de Potier de MS2 fournies par les deux méthodes.

\begin{tabular}{|l|c|c|c|}
\cline { 2 - 4 } \multicolumn{1}{c|}{} & \multicolumn{2}{c|}{ Nouvelle méthode (ksat=6; formules 15.2) } & \multirow{2}{*}{ Méthode classique } \\
\hline Points & $\mathbf{A}_{\mathbf{d w}}$ & $\mathbf{B}_{\mathrm{dw}}$ & \\
\hline $\mathrm{I}(\mathbf{A})$ & 1 & 1.9 & 137 \\
\hline $\mathbf{V}(\mathbf{V})$ & 162.5 & 161 & 1,3242 \\
\hline$\lambda \omega(\Omega)$ & 1,3548 & 1,4261 & 0,3548 \\
\hline$\alpha$ & 0,3604 & 0,3555 & \\
\hline
\end{tabular}

Les résultats prouvent une fois de plus, la justesse de notre approche puisque les deux méthodes fournissent des valeurs sensiblement identiques. Au niveau des valeurs du paramètre $\lambda \omega$, l'écart entre les résultats des deux méthodes est d'autant réduit que le courant d'induit de l'essai en déwatté de notre méthode est faible (point $A_{d w}$ ).

Pour confirmer la validité de la méthode analytique proposée, nous avons relevé lors d'un essai sur charge inductive, le quadruplet $\left(\mathrm{V}, \mathrm{I}, \mathrm{J}_{\mathrm{e} 1}, \varphi\right)$ correspondant à deux points de fonctionnement différents. Ensuite, en utilisant les valeurs des paramètres de la table 8 , nous avons calculé à l'aide des équations de Potier, la valeur du courant d'excitation $J_{\mathrm{e} 1}$ nécessaire pour obtenir le triplet $(V, I, \varphi)$ des charges précédentes. Les valeurs obtenues ont été ensuite comparées dans la table 9 à celles mesurées.

Table 9 : Mesure et prédétermination des courants d'excitation de deux points de fonctionnement de MS2

\begin{tabular}{|c|c|c|}
\cline { 3 - 4 } Mesures & \multicolumn{2}{|c|}{ Calculs } \\
\cline { 2 - 3 } & Nouvelle méthode & Méthode \\
\hline
\end{tabular}




\begin{tabular}{|c|c|c|c|c|c|c|c|c|c|c|}
\hline \multirow[b]{2}{*}{ Grandeurs } & \multirow[b]{2}{*}{$\mathrm{U}(\mathrm{V})$} & \multirow[b]{2}{*}{$I(A)$} & \multirow[b]{2}{*}{$J_{e 1}(A)$} & \multirow[b]{2}{*}{$\cos \varphi$} & \multicolumn{2}{|c|}{$A_{d w}$} & \multicolumn{2}{|c|}{$B_{d w}$} & \multicolumn{2}{|c|}{ classique } \\
\hline & & & & & $J_{\mathrm{e} 1}(A)$ & $\left|\varepsilon_{\mathrm{r}}\right|(\%)$ & $\mathrm{J}_{\mathrm{e} 1}(\mathrm{~A})$ & $\left|\varepsilon_{\mathrm{r}}\right|(\%)$ & $\mathrm{J}_{\mathrm{e} 1}(\mathrm{~A})$ & $\left|\varepsilon_{r}\right|(\%)$ \\
\hline Point 1 & 219 & 13,8 & 8,7 & 0,7 & 8,8547 & $1,78 \%$ & 8,7986 & 1,13 & 8,7779 & $0,9 \%$ \\
\hline Point 2 & 190 & 13,8 & 7,8 & 0,61 & 7,7999 & $0 \%$ & 7,8339 & 0,43 & 7,7219 & $1 \%$ \\
\hline
\end{tabular}

Comme nous pouvons le constater, les valeurs de $\mathrm{J}_{\mathrm{e} 1}$ calculées sont très proches de celles mesurées et notre méthode reste performante même pour le point $B_{d w}$ de courant d'induit plus important (environ $12,5 \%$ de la valeur nominale). Nous suggérons toutefois en pratique, de ne pas dépasser $10 \%$ de la valeur nominale du courant d'induit conformément à notre approche qui est fondée sur un courant d'induit faible.

\section{Conclusion}

Nous avons présenté dans cet article une nouvelle méthode de détermination des paramètres de Potier, fondée sur les mêmes essais (essais à vide, en court-circuit et en déwatté) que la méthode classique mais utilisant une approche différente. Cette méthode qui est purement analytique, propose des formules de calcul des paramètres de Potier dans lesquelles interviennent les pentes des droites associées aux différentes caractéristiques relevées lors des essais. La mise en œuvre de la méthode nécessite le relevé d'un nombre limité de points ( 5 points suffisent) et les puissances mises en jeu lors de l'essai en déwatté, sont réduites du fait de la réalisation de l'essai sous faible courant d'induit (inférieur à $10 \%$ du courant nominal).

Les expérimentations entreprises ont confirmé la validité de la méthode proposée. Les résultats obtenus sont comparables, voire meilleurs, à ceux de la méthode classique, qui est sujette à des erreurs de construction et qui parfois, conduit à des indéterminations. Par souci d'une plus grande souplesse dans la détermination pratique des paramètres de Potier, nous préconisons pour notre méthode, d'adopter les formules simplifiées (15.2) ( table 1) qui ont été obtenues en négligeant la résistance statorique. 


\section{Bibliographie}

[1] E. Potolea, S. Patracoiu, Le modèle mathématique de la machine synchrone dans le régime transitoire quasi sinusoïdal, $\mathrm{RGE}-\mathrm{N}^{\circ}$ 1/91, 1991.

[2] S.-A. Tahan, I. Kamwa, P. Viarouge, M. Ferfra, A generalized model of saturated synchronous machines, Mathematics and Computers in simulation, Volume 38, Issues 4-6, pp. 423-435, August 1995.

[3] G. Sturtzer, E. Smiguiel, Modélisation et commande des moteurs triphasés, TECHNO-SUP, Ellipses, 2000.

[4] H. Ben Ahmed, N. Bernard, G. Feld, B. Multon, Machines synchrones - Modélisation en régime permanent, Techniques de l'ingénieur, D 3 521, 11/2006.

[5] G. Seguier, F. Notelet, Electrotechnique Industrielle, $2^{\text {ème }}$ Edition, Paris, Technique \& Documentation - Lavoisier, 1994.

[6] Dalmasso J.L., Cours d'Electrotechnique, (Tome 2) machines tournantes à courants alternatifs, Paris, Belin, 1985.

[7] Parker F., Alternating Current Generators, Electrical Engineer's Reference Book (Sixteenth Edition), pp. 1-59, 2003. 